\title{
A Two-stage Method for Inverse Medium Scattering
}

\author{
Kazufumi Ito* $\quad$ Bangti $\mathrm{Jin}^{\dagger} \quad$ Jun Zou ${ }^{\ddagger}$
}

October 31, 2018

\begin{abstract}
We present a novel numerical method to the time-harmonic inverse medium scattering problem of recovering the refractive index from near-field scattered data. The approach consists of two stages, one pruning step of detecting the scatterer support, and one resolution enhancing step with mixed regularization. The first step is strictly direct and of sampling type, and faithfully detects the scatterer support. The second step is an innovative application of nonsmooth mixed regularization, and it accurately resolves the scatterer sizes as well as intensities. The model is efficiently solved by a semi-smooth Newton-type method. Numerical results for two- and three-dimensional examples indicate that the approach is accurate, computationally efficient, and robust with respect to data noise.

Key words: inverse medium scattering problem, reconstruction algorithm, sampling method, mixed regularization, semi-smooth Newton method
\end{abstract}

\section{Introduction}

In this work we study the inverse medium scattering problem (IMSP) of determining the refractive index from near-field measurements for time-harmonic wave propagation [8]. Consider a homogeneous background space $\mathbb{R}^{\mathrm{d}}(\mathrm{d}=2,3)$ that contains some inhomogeneous media occupying a bounded domain $\Omega$. Let $u^{i n c}=e^{i k x \cdot d}$ be an incident plane wave, with the incident direction $d \in \mathbb{S}^{d-1}$ and the wave number $k$. Then the total field $u$ induced by the inhomogeneous medium scatterers satisfies the Helmholtz equation [8]

$$
\Delta u+k^{2} n^{2}(x) u=0
$$

where the function $n(x)$ is the refractive index, i.e., the ratio of the wave speed in the homogeneous background to that in the concerned medium at location $x$. The model describes not only time-harmonic acoustic wave propagation, but also electromagnetic wave propagation in either the transverse magnetic or transverse electric modes [16, Appendix].

Next we let $\eta=\left(n^{2}-1\right) k^{2}$, which combines the relative refractive index $n^{2}-1$ with the wave number $k$. Clearly the coefficient $\eta$ characterizes the material properties of the inhomogeneity

\footnotetext{
* Department of Mathematics and Center for Research in Scientific Computation, North Carolina State University, Raleigh, North Carolina (kito@unity.ncsu.edu).

${ }^{\dagger}$ Department of Mathematics and Institute for Applied Mathematics and Computational Science, Texas A\&M University, College Station, Texas 77843-3368, USA (btjin@math.tamu.edu).

${ }^{\ddagger}$ Department of Mathematics, Chinese University of Hong Kong, Shatin, N.T., Hong Kong (zou@math.cuhk.edu.hk).
} 
and is supported in the scatterer $\Omega \subset \mathbb{R}^{\mathrm{d}}$. We denote by $I=\eta u$ the induced current, by $G(x, y)$ the fundamental solution to the Helmholtz equation in the homogeneous background, i.e.,

$$
G(x, y)=\left\{\begin{aligned}
\frac{i}{4} H_{0}^{1}(k|x-y|), & \mathrm{d}=2, \\
\frac{1}{4 \pi} \frac{e^{i k|x-y|}}{|x-y|}, & \mathrm{d}=3,
\end{aligned}\right.
$$

where the function $H_{0}^{1}$ refers to Hankel function of the first kind and zeroth-order. Then we can express the total field $u$ as follows [8]

$$
u=u^{i n c}+\int_{\Omega} G(x, y) I(y) d y
$$

By multiplying both sides of equation (2) by $\eta$, we arrive at the following integral equation of the second kind for the induced current $I$

$$
I(x)=\eta u^{i n c}+\eta \int_{\Omega} G(x, y) I(y) d y .
$$

The reformulation (3) is numerically amenable since all computation is restricted to the scatterer support $\Omega$, which is much smaller than the whole space $\mathbb{R}^{\mathrm{d}}$. Hence, the complexity is also very low. We will approximate the solution to (3) by the mid-point rule (cf. Appendix A).

Next we let $u^{s}=u-u^{i n c}$ be the scattered field, which is measured on a closed curve/surface $\Gamma$ enclosing the scatterers $\Omega$. Then the IMSP is to retrieve the refractive index $n^{2}$ or equivalently the coefficient $\eta$ from (possibly very noisy) measurements of the scattered field $u^{s}$, corresponding to one or several incident fields. In the literature, a number of reconstruction techniques for the IMSP have been developed. These methods can be roughly divided into two groups: support detection and coefficient estimate. The former group (including MUSIC [10,6], linear sampling method [7,3] and factorization method [20] etc.) usually is of sampling type, and aims at detecting the scatterer support efficiently. The latter group generally relies on the idea of regularization (including Tikhonov regularization [2, 23], iterative regularization method [12, 1, 13], contrast source inversion [27], subspace regularization [5] and propagation-backpropagation method [28]), and aims at retrieving a distributed estimate of the index function. These approaches generally are more expensive, but their results may profile the inhomogeneities more precisely.

In this paper, we shall develop a novel two-stage numerical method for solving the IMSP. The first step employs a direct sampling method, recently developed in [16], to detect the scatterer support $\Omega$ stably and accurately. It is based on the following index function

$$
\Phi\left(x_{p}\right)=\frac{\left|\left\langle u^{s}, G\left(\cdot, x_{p}\right)\right\rangle_{L^{2}(\Gamma)}\right|}{\left\|u^{s}\right\|_{L^{2}(\Gamma)}\left\|G\left(\cdot, x_{p}\right)\right\|_{L^{2}(\Gamma)}} \quad \forall x_{p} \in \widetilde{\Omega},
$$

where $\widetilde{\Omega} \supset \Omega$ is a sampling domain. Numerically, the method is strictly direct and does not incur any linear matrix operations. The method can detect reliably the scatterer support $\Omega$ even in the presence of a large amount of data noises [16]. In particular, a (much smaller) computational domain $D \subset \widetilde{\Omega}$ can be determined from the index $\Phi$, and furthermore the restriction $\left.\Phi\right|_{D}$ may serve as a first approximation to the coefficient $\eta$.

The second step enhances the image resolution by a novel application of (nonsmooth) mixed regularization. With the approximation $\left.\Phi\right|_{D}$ from the sampling step at hand, equation (3) gives 
an approximate induced current $\widehat{I}$ as well as an approximate total field $\widehat{u}$. Then we seek a regularized solution $\eta$ to the linearized scattering equation

$$
\int_{D} G(x, y) \widehat{u}(y) \eta(y) d y=u^{s}(x),
$$

by an innovative regularization incorporating both $L^{1}$ and $H^{1}$ penalties. The $L^{1}$ penalty promotes the sparsity of the solution $[25,4,11]$. However, the estimate tends to be very spiky with the $L^{1}$ penalty used alone. Meanwhile, the conventional $H^{1}$ penalty can only yield globally smooth but often overly diffusive profiles. In this work we shall propose a novel mixed model that consists of a suitable combination of the $L^{1}$ and $H^{1}$ penalties. As we will see, this mixed model produces well clustered and yet distributed solutions, thereby overcoming the aforementioned drawbacks. It is the mixed model that enables us to obtain a clear and accurate reconstruction of the inclusions: The homogeneous background is vividly separated from the scatterers and both support and intensity of the inclusions are accurately resolved.

Numerically, the $L^{1}$ penalty term gives rise to a nonsmooth optimality condition, which renders its direct numerical treatment inconvenient. Fortunately, by using complementarity functions, the optimality condition reduces to a coupled nonlinear system for the sought-for coefficient $\eta$ and the Lagrangian multiplier, which is amenable to efficient numerical solution. We shall develop an efficient and stable semi-smooth Newton solver for the model via a primaldual active-set strategy [18]. Overall, the direct sampling method is very cheap and reduces the computational domain $D$ in the mixed model (cf. (5)) significantly, which in turn makes the semi-smooth Newton method for the mixed model very fast. Hence, the proposed inverse scattering method is computationally very efficient.

The rest of the paper is structured as follows. In Section 2, we recall a novel direct sampling method for screening the scatterer support $\Omega$, and derive thereby an initial guess to the coefficient $\eta$. Then in Section 3 we develop an enhancement technique based on the idea of mixed regularization, and an efficient semi-smooth Newton solver. Finally, we present numerical results for two- and three-dimensional examples to demonstrate the accuracy and efficiency of the proposed inverse scattering method.

\section{$2 \quad$ A direct sampling method}

In this section, we describe a direct sampling method to determine the shape of the scatterers, recently derived in [16]. We only briefly recall the derivation, but refer the readers to [16] for more details. Consider a circular curve $\Gamma(d=2)$ or a spherical surface $\Gamma(d=3)$. Let $G\left(x, x_{p}\right)$ be the fundamental solution in the homogeneous background. Then using the definitions of the fundamental solutions $G\left(x, x_{p}\right)$ and $G\left(x, x_{q}\right)$ and Green's second identity, we deduce

$$
2 i \Im\left(G\left(x_{p}, x_{q}\right)\right)=\int_{\Gamma}\left[\bar{G}\left(x, x_{q}\right) \frac{\partial G\left(x, x_{p}\right)}{\partial n}-G\left(x, x_{p}\right) \frac{\partial \bar{G}\left(x, x_{q}\right)}{\partial n}\right] d s,
$$

where the points $x_{p}, x_{q} \in \Omega_{\Gamma}$, the domain enclosed by the boundary $\Gamma$.

Next we approximate the right hand side of identity (6) by means of the Sommerfeld radiation condition for the Helmholtz equation, i.e.,

$$
\frac{\partial G\left(x, x_{p}\right)}{\partial n}=i k G\left(x, x_{p}\right)+\text { h.o.t. }
$$


Consequently, we arrive at the following approximate relation

$$
\int_{\Gamma} G\left(x, x_{p}\right) \bar{G}\left(x, x_{q}\right) d s \approx k^{-1} \Im\left(G\left(x_{p}, x_{q}\right)\right),
$$

which is valid if the points $x_{p}$ and $x_{q}$ are not close to the boundary $\Gamma$.

Now, we consider a sampling domain $\widetilde{\Omega}$ enclosing the scatterer support $\Omega$. Upon dividing $\widetilde{\Omega}$ into small elements $\left\{\tau_{j}\right\}$, we may approximate the integral in the scattering relation (2) by

$$
u^{s}(x)=\int_{\widetilde{\Omega}} G(x, y) I(y) d y \approx \sum_{j} w_{j} G\left(x, y_{j}\right),
$$

where the weight $w_{j}$ is given by $\left|\tau_{j}\right| I_{j}$ with $\left|\tau_{j}\right|$ being the volume of the $j$ th element $\tau_{j}$. The relation (7) is plausible if the induced current $I$ is regular in each element and the elements $\left\{\tau_{j}\right\}$ are sufficiently fine. It also admits a nice physical interpretation: the scattered field $u^{s}$ at any fixed point $x \in \Gamma$ is a weighted average of that due to point scatterers located at $\left\{y_{j}\right\}$.

Combining the preceding two relations yields

$$
\int_{\Gamma} u^{s}(x) \bar{G}\left(x, x_{p}\right) d s \approx k^{-1} \sum_{j} w_{j} \Im\left(G\left(y_{j}, x_{p}\right)\right) .
$$

Hence, if the sampling point $x_{p}$ is close to some point scatterer $y_{j}$, i.e., $y_{j} \in \Omega$, then $G\left(y_{j}, x_{p}\right)$ is nearly singular and takes a very large value, contributing significantly to the sum in (8). Conversely, if the point $x_{p}$ is far away from all the physical point scatterers, then the sum will be very small due to the decay property of $G\left(y_{j}, x_{p}\right)$.

These facts lead us to the index function $\Phi\left(x_{p}\right)$ in (4) for any $x_{p}$ in the sampling region $\widetilde{\Omega}$. In practice, if a point $x_{p}$ satisfies $\Phi\left(x_{p}\right) \approx 1$, then it likely lies within the support; whereas if $\Phi\left(x_{p}\right) \approx 0$, then the point $x_{p}$ most probably lies outside the support. Hence it serves as an indicator of the scatterer support. Consequently, we can determine a domain $D \subset \widetilde{\Omega}$ as one approximate scatterer support, and moreover, the restriction $\left.\Phi\right|_{D}$ of the index $\Phi$ to the domain $D$ may be regarded as a first approximation to the sought-for coefficient $\eta$. The subdomain $D$ may be chosen as $D=\left\{x \in \widetilde{\Omega}: \Phi(x) \geq \mu \max _{x \in \widetilde{\Omega}} \Phi(x)\right\}$ with $\mu$ being a cut-off value, i.e., the union of elements whose index values are not less than a specified fraction of the largest index value over the sampling region $\widetilde{\Omega}$. This determination of subdomain $D$ will be adopted in our numerical experiments.

This method is of sampling type (cf. [22] for an overview of existing sampling methods), and its flavor closely resembles multiple signal classification $[24,6,10]$ and linear sampling method $[7,20]$. However, unlike these existing techniques, it works with a few (e.g., one or two) incident waves, is highly tolerant with respect to noise, and involves only computing inner products with fundamental solutions rather than expensive matrix operations as in the other two techniques. The robustness of $\Phi$ is attributed to the fact that the (high-frequency) noise is roughly orthogonal to the (smooth) fundamental solutions.

\section{Mixed regularization}

The direct sampling method in Section 2 extracts an accurate estimate $D$ to the scatterer support $\Omega$ as well as a reasonable initial guess to the medium coefficient $\eta$, i.e., $\left.\Phi\right|_{D}$. In this part we 
refine the approximation $\left.\Phi\right|_{D}$ by exploiting the idea of nonsmooth mixed regularization. Given the approximation $\left.\Phi\right|_{D}$, we can compute the induced current $\widehat{I}$ via (3) for each incident wave and the corresponding total field $\widehat{u}$ on the domain $D$ from (2). By substituting the approximation $\widehat{u}$ into equation (2), we arrive at the following linearized problem

$$
\int_{D} G(x, y) \widehat{u}(y) \eta(y) d y=u^{s}(x), \quad x \in \Gamma .
$$

It is convenient to introduce a linear integral operator $K: L^{2}(D) \mapsto L^{2}(\Gamma)$ defined by

$$
(K \eta)(x)=\int_{D} G(x, y) \widehat{u}(y) \eta(y) d y .
$$

We observe that the kernel $G(x, y) \widehat{u}(y)$ is smooth due to the analyticity of fundamental functions $G(x, y)$ away from the singularities and standard Sobolev smoothness of the total field $\widehat{u}(y)$ (following from elliptic regularity theory [8]). Hence, the linear operator $K: L^{2}(D) \mapsto L^{2}(\Gamma)$ is compact. As a consequence, the linearized problem (9) is ill-posed in the sense that small perturbations in the data can lead to huge deviations in the solution, according to the classical inverse theory [26], which is reminiscent of the severe ill-posedness of the IMSP, and its stable and accurate numerical solution calls for regularization techniques.

We determine an enhanced estimate of the coefficient $\eta$ from the linearized problem (9) by solving the following variational problem:

$$
\min \frac{1}{2} \int_{\Gamma}\left|K \eta-u^{s}\right|^{2} d s+\alpha \int_{D}|\eta| d x+\frac{\beta}{2} \int_{D}|\nabla \eta|^{2} d x
$$

In comparison with more conventional regularization techniques, the most salient feature of the model (10) lies in two penalty terms: it contains both the $L^{1}$ penalty and the $H^{1}$ penalty, which exert drastically different a priori knowledge of the sought-for solution. The scalars $\alpha$ and $\beta$ are regularization parameters controlling the strength of respective regularization.

This variational problem (10) allows us to determine a coefficient $\eta$ which is distributed yet clustered, i.e., exhibiting a clear groupwise sparsity structure in the canonical pixel basis. This a priori knowledge is plausible for localized inclusions/inhomogeneities in a homogeneous background. The model (10) is derived from the following widely accepted observations. The $L^{1}$ penalty promotes the sparsity of the solution [25, 4, 11], i.e., the solution is very much localized. Hence, the estimated background is homogeneous. However, if the $L^{1}$ penalty is used alone, the solution tends to be very spiky and may miss numerous physically relevant pixels in the soughtfor groups. That is, the desirable groupwise structure is not preserved. Meanwhile, the more conventional $H^{1}$ penalty [26] yields a globally smooth profile, but the solution is often overly diffusive. Consequently, the overall structure stands out clearly, but the retrieved background is very blurry, which may lead to erroneous diagnosis of the number of the inclusions and their sizes. In order to preserve simultaneously these distinct features of the sought-for coefficient, i.e., sparsely distributed groupwise structures in a homogeneous background, a natural idea would be to combine the $L^{1}$ penalty with the $H^{1}$ penalty, in the hope of retaining the strengths of both models. As we shall see below, the idea does work very well, and the model is very effective for enhancing the resolution of the estimate to the coefficient $\eta$.

The general idea of mixed regularization, i.e., using multiple penalties, has proved very effective in promoting several distinct features simultaneously. This general idea has been pursued 
in the imaging community $[17,21]$. However, to the best of our knowledge, the model (10) has not been explored in the literature, let alone its efficient and accurate numerical treatment. A detailed mathematical analysis of the model (10) is beyond the scope of the present paper. We refer interested readers to [14] for some preliminary results on mixed regularization and to [19] for a related model (elastic-net).

To fully explore the potentials of the model (10), an efficient and accurate solver is required. We shall develop a semi-smooth Newton type method, which allows extracting very detailed features of the solutions to the model (10). The starting point of the algorithm is the necessary optimality condition of the variational problem (10), which reads

$$
K^{*} K \eta-\beta \Delta \eta-K^{*} u^{s} \in-\alpha \partial \psi(\eta)
$$

where $\psi(\eta)=\|\eta\|_{L^{1}}$ and the subdifferential $\partial \psi(\eta)[18]$ is the set-valued signum function, which is defined pointwise as

$$
\partial \psi(\eta)(x)= \begin{cases}1, & \text { if } \eta(x)>0 \\ {[-1,1],} & \text { if } \eta(x)=0 \\ -1, & \text { if } \eta(x)<0\end{cases}
$$

Due to the convexity of the functional, the relation (11) is also a sufficient condition. Hence it suffices to solve the inclusion (11), for which there are several different ways, e.g., iterative soft shrinkage [9, 29], augmented Lagrangian method/alternating direction method or semi-smooth Newton method [18]. We shall develop a (new) semi-smooth Newton method to efficiently solve the inclusion (11). To this end, we first recall the complementarity condition [18]

$$
\lambda=\frac{\lambda+c \eta}{\max (1,|\lambda+c \eta|)}
$$

for any $c>0$, which will be fixed at a constant in the implementation, and $\lambda$ serves as a Lagrange multiplier. It can be directly verified by pointwise inspection that the complementarity condition (12) is equivalent to the inclusion $\lambda \in \partial \psi(\eta)$ (cf. [18]). With the help of the complementarity condition (12), we arrive at the following equivalent nonlinear system in the primal variable $\eta$ and dual variable $\lambda$ :

$$
\left\{\begin{aligned}
K^{*} K \eta-\beta \Delta \eta-K^{*} u^{s}+\alpha \lambda & =0, \\
\lambda-\frac{\lambda+c \eta}{\max (1,|\lambda+c \eta|)} & =0 .
\end{aligned}\right.
$$

Then we apply the semi-smooth Newton algorithm using a primal-dual active set strategy. The complete implementation is listed in Algorithm 1. The technical details for deriving the crucial Newton step (Step 5) are deferred to Appendix B, which involves damping and regularization. A natural choice of the stopping criterion at Step 6 is based on monitoring the change of the active set $\mathcal{A}=\{x \in D:|\lambda+c \eta| \leq 1\}$ : if the active sets for two consecutive iterations coincide, then we can terminate the algorithm, cf. [18].

The main computational effort of the algorithm lies in the Newton update at Step 5: each iteration requires solving a (dense) linear system. We note that the dual variable $\lambda$ can be expressed in terms of the primal variable $\eta$ and on the active set $\mathcal{A}$, the coefficient $\eta$ vanishes identically. Thus in practice, we solve only a linear system for $\eta$ on the inactive set $\mathcal{I}=D \backslash \mathcal{A}$. An important feature of the algorithm is that the linear system becomes smaller and smaller and also less and less ill-conditioned as the iteration goes on, while the iterate captures more and more refined details of the nonhomogeneous medium regions. If the exact solution is indeed 


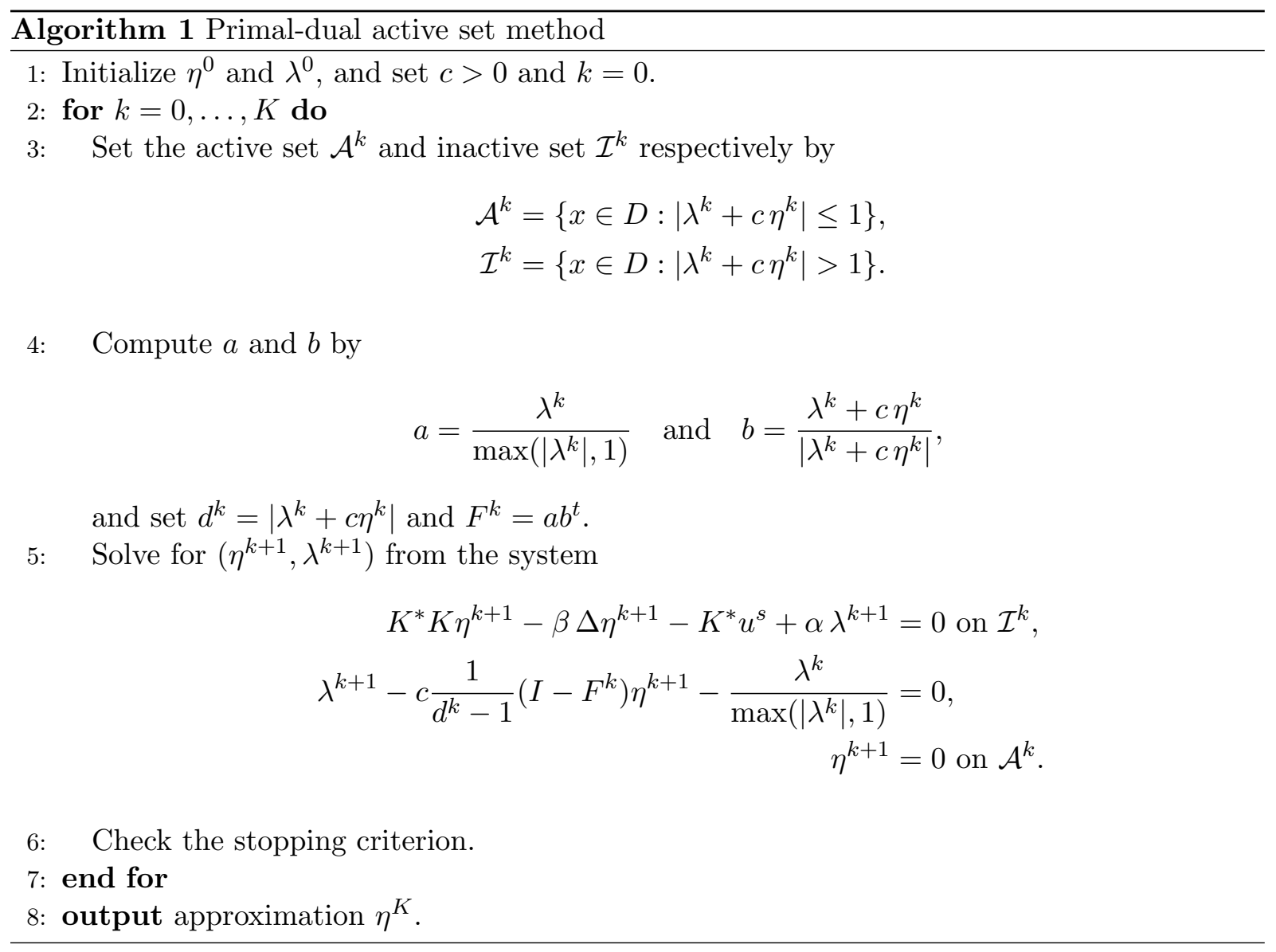

sparse (many zero entries), then the system size, i.e., $|\mathcal{I}|$, usually shrinks quickly as the iteration proceeds. The numerical experiments indicate that the convergence of the algorithm is rather steady and fast.

\section{$4 \quad$ Numerical experiments}

In this part, we present numerical results for several two- and three-dimensional examples to showcase the proposed two-stage inverse scattering method, for both exact and noisy data. The wave number $k$ is fixed at $2 \pi$, and the wavelength is set to $\lambda=1$. The exact scattered field $u^{s}$ is obtained by first solving the integral equation (3) for the induced current $I$ and then substituting the current $I$ into the integral representation (2). Here the integral equation (3) is discretized by a mid-point rule; see Appendix A for details. The noisy scattered data $u_{\delta}^{s}$ are generated pointwise by the formula

$$
u_{\delta}^{s}(x)=u^{s}(x)+\epsilon \zeta \max _{x \in \Gamma}\left|u^{s}(x)\right|,
$$

where $\epsilon$ refers to the relative noise level, and both the real and imaginary parts of the noise $\zeta=\zeta(x)$ follow the standard Gaussian distribution. The index $\Phi$, its restriction $\left.\Phi\right|_{D}$ and the enhanced approximation $\eta$ by the mixed model will be displayed. As is mentioned in Section 2, we choose the subdomain $D$ (approximate scatterer support) based on the formula $D=\{x \in$ 

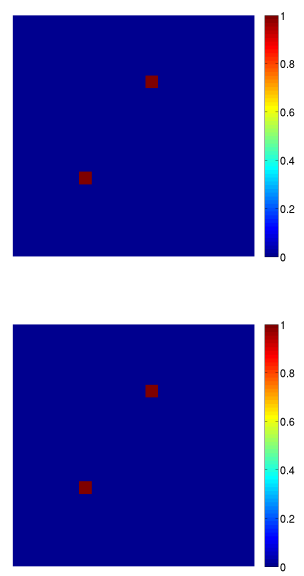

(a) true scatterer
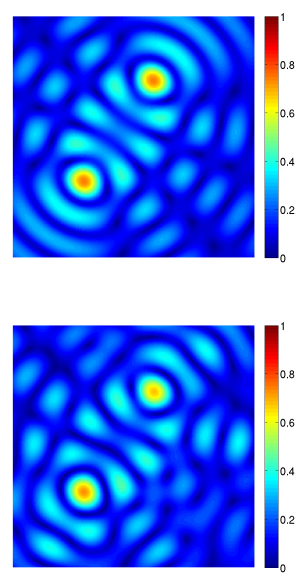

(b) index $\Phi$
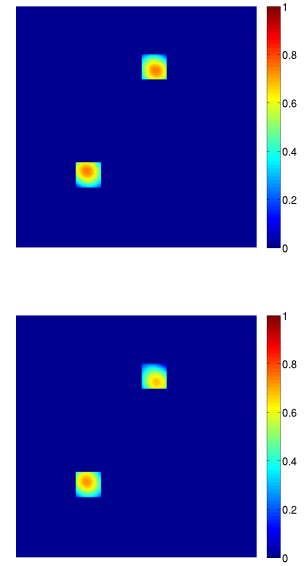

(c) index $\left.\Phi\right|_{D}$
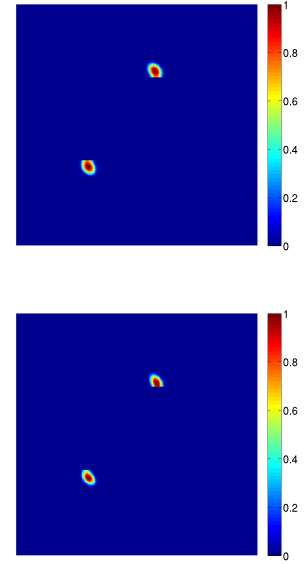

(d) sparse recon.

Figure 1: Numerical results for Example 1(a): (a) true scatterer, (b) index $\Phi$, (c) index $\left.\Phi\right|_{D}$ (restriction to the subdomain $D$ ) and (d) sparse reconstruction. The first and second rows refer to exact data and the data with $20 \%$ noise, respectively.

$\left.\widetilde{\Omega}: \Phi(x) \geq \mu \max _{x \in \widetilde{\Omega}} \Phi(x)\right\}$, where the cut-off value $\mu$ is taken in the range $(0.5,0.7)$. The choice of the cutoff value $\mu$ affects directly the size of the domain $D$, but does not cause much effects on the reconstructions.

Like in any regularization technique, an appropriate choice of regularization parameters $(\alpha, \beta)$ in the mixed model (10) is crucial for the success of the proposed imaging algorithm. There have been a number of choice rules [15] for one single parameter, but very little is known about the mixed model. We shall choose the pair $(\alpha, \beta)$ in a trial and error manner, which suffices our goal of illustrating the significant potentials of the mixed model for inverse scattering. In Algorithm 1, the parameter $c$ is set to 50, and both $\eta^{0}$ and $\lambda^{0}$ are initialized to zero. The maximum number $K$ of Newton iterations is 50. In all the experiments, the convergence of the algorithm is achieved within about 10 iterations. All the computations were performed on MATLAB 7.12.0 (R2011a) on a dual-core desktop computer with 2GB RAM.

\subsection{Two-dimensional examples}

Unless otherwise specified, one incident direction $d$ is employed for two-dimensional problems, and it is fixed at $\frac{1}{\sqrt{2}}(1,1)^{\mathrm{T}}$. The scattered field $u^{s}$ is measured at 30 points uniformly distributed on a circle of radius 5 . The sampling domain $\widetilde{\Omega}$ is fixed at $[-2,2]^{2}$, which is divided into a uniform mesh consisting of small squares of width $h=0.01$. The subdomain $D$ for the integral equation (9) is divided into a coarser uniform mesh consisting of small squares of width 0.02.

Our first example illustrates the method for two separate scatterers.

Example 1. We consider two separate square scatterers in the following two scenarios

(a) The scatterers are of width 0.2 and centered at $(-0.8,-0.7)$ and $(0.3,0.9)$, respectively, and the coefficient $\eta$ in both region is 1. 
Table 1: Regularization parameters $(\alpha, \beta)$ for the examples.

\begin{tabular}{ccccc}
\hline example & $1(\mathrm{a})$ & $1(\mathrm{~b})$ & 2 & 3 \\
\hline$\epsilon=0 \%$ & $(2.0 \mathrm{e}-6,1.5 \mathrm{e}-9)$ & $(8.0 \mathrm{e}-6,1.4 \mathrm{e}-8)$ & $(7.0 \mathrm{e}-6,1.0 \mathrm{e}-9)$ & $(2.5 \mathrm{e}-9,4.0 \mathrm{e}-14)$ \\
$\epsilon=20 \%$ & $(3.0 \mathrm{e}-6,2.0 \mathrm{e}-9)$ & $(8.5 \mathrm{e}-6,9.0 \mathrm{e}-9)$ & $(7.0 \mathrm{e}-6,5.0 \mathrm{e}-9)$ & $(2.5 \mathrm{e}-9,5.0 \mathrm{e}-14)$ \\
\hline
\end{tabular}

(b) The scatterers are of width 0.3 and centered at $(-0.25,0)$ and $(0.25,0)$, respectively, and the coefficient $\eta$ in the former and latter is 1.5 and 1 , respectively.

The two scatterers in Example 1(a) are well apart from each other. The recovery of the scatterer locations by the index $\Phi$ is quite satisfactory, especially upon noting that we have just used one incident wave. Two distinct scatterers are observed for both exact data and the data with $20 \%$ noise, cf. Fig. 1(b). However, the magnitudes are inaccurate, and the estimate suffers from spurious oscillations in the homogeneous background, due to the ill-posed nature of the IMSP and the oscillating behavior of fundamental solutions. Nonetheless, two localized square subregions $D$ (each of width 0.4 ) encompass the modes of the index $\Phi$, see Fig. 1(c), and may be taken as an approximate scatterer support. In Fig. 1(c), the entire sampling domain $\widetilde{\Omega}$ is shown, and the two small squares represent the approximate support $D$. Outside of the domain $D$, the index $\left.\Phi\right|_{D}$ is set to zero, i.e., identical with homogeneous background, and will not be updated during the enhancing step. The enhancing step is initialized with $\left.\Phi\right|_{D}$, and the results are shown in Fig. 1(d). The regularization parameters for getting the reconstructions, which are determined in a trial-and-error manner, are presented in Table 1. The enhancement of the approximation $\Phi_{D}$ over the domain $D$ is significant: the recovered background is now mostly homogeneous, and the magnitudes and sizes of the recovered scatterers agree well with the exact ones. This shows clearly the significant potentials of the proposed mixed regularization for inverse scattering problems. The numbers in Table 1 also sheds valuable insights into the mixed model (10): the value of the regularization parameter $\alpha$ is much larger than that of $\beta$. Hence, the $L^{1}$ penalty plays a predominant role in ensuring the sparsity of the solution, whereas the $H^{1}$ penalty yields a locally smooth structure.

The two scatterers in Example 1(b) stay very close to each other, and thus it is rather challenging for precise numerical reconstruction. The detection of the scatterer locations by the index $\Phi$, see Fig. 2(b), is still very impressive. In particular, it clearly distinguishes two separate scatterers with their locations correctly retrieved, and this remains stable for data with up to $20 \%$ noise. The mixed regularization is performed on a square $D$ (of width 1 ) enclosing the two modes in the index $\Phi$, see Fig. 2(c). This choice of the inversion domain $D$ allows possibly connecting of the modes. However, the enhancement correctly recognizes two separate scatterers, with their magnitudes and sizes in excellent agreement with the exact ones. Also the estimated background is very crispy. Surprisingly, the estimate deteriorates only slightly in that the right scatterer elongates a little bit towards the left scatterer as the noise level $\epsilon$ increases from 0 to $20 \%$. Although not presented, we would like to note that for this particular example, the reconstructions are still reasonable for data with $30 \%$ noise. Hence the proposed inverse scattering method is very robust with respect to the data noise.

Next we consider a ring-shaped scatterer.

Example 2. The scatterer is one ring-shaped square located at the origin, with the outer and inner side lengths being 0.6 and 0.4 , respectively. The coefficient $\eta$ of the scatterer is 1 . Two incident directions $d_{1}=\frac{1}{\sqrt{2}}(1,1)^{\mathrm{T}}$ and $d_{2}=\frac{1}{\sqrt{2}}(1,-1)^{\mathrm{T}}$ are considered. 

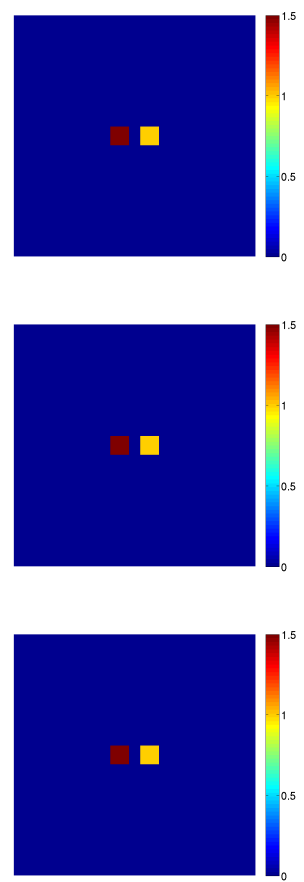

(a) true scatterer
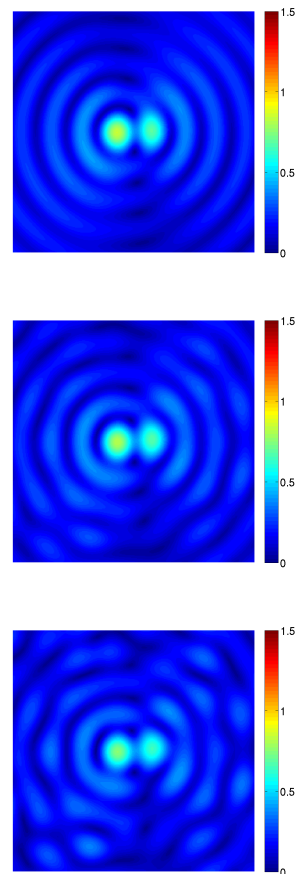

(b) index $\Phi$
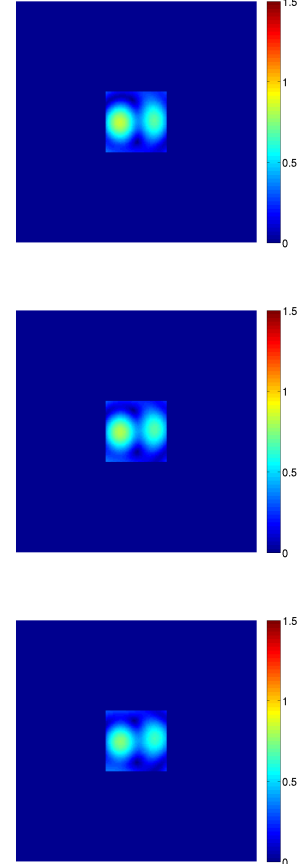

(c) index $\left.\Phi\right|_{D}$
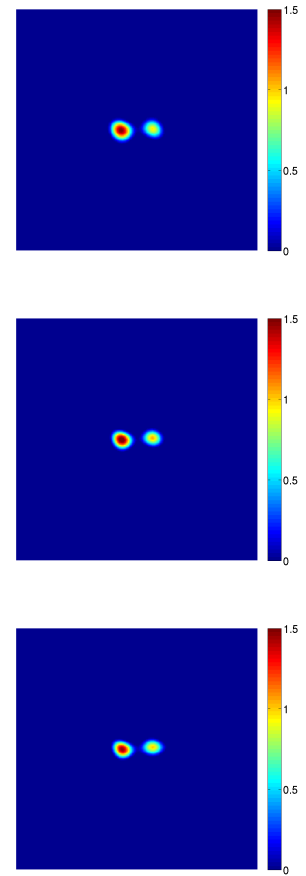

(d) sparse recon.

Figure 2: Numerical results for Example 1(b): (a) true scatterer, (b) index $\Phi$, (c) index $\left.\Phi\right|_{D}$ (restriction to the subdomain $D$ ) and (d) sparse reconstruction. From top to bottom, the rows refer to exact data, noisy data with $10 \%$ and $20 \%$, respectively.

Ring-shaped scatterer represents one of most challenging objects to recover, and it is highly nontrivial even with multiple scattered field data sets, especially noting the ring has a small thickness. It has been observed that one single incident field is insufficient to completely resolve the ring structure, and only some parts of the ring can be resolved, depending on the incident direction $d$ [16]. Hence we employ two incident waves in the directions $d_{1}=\frac{1}{\sqrt{2}}(1,1)^{\mathrm{T}}$ and $d_{2}=\frac{1}{\sqrt{2}}(1,-1)^{\mathrm{T}}$ in order to yield sufficient amount of information about the scatterer, and accordingly, the index function $\Phi$ is defined as follows

$$
\Phi\left(x_{p}\right)=\max _{i}\left\{\Phi_{i}\left(x_{p}\right)\right\} \quad \forall x_{p} \in \widetilde{\Omega},
$$

where the function $\Phi_{i}$ refers to the index for the $i$ th data set. The numerical results with the exact data and $20 \%$ noise in the data are shown in Fig. 3. With just two incident waves, the index $\Phi$ can provide a quite reasonable estimate of the ring shape. Despite some small oscillations, the overall profile stands out clearly, and remains very stable for up to $20 \%$ noise in the data. The enhancing step via mixed regularization provides a very crispy estimate of the ring structure: the recovered scatterer has a clear ring structure, which agrees excellently with the exact one in terms of both magnitude and size. The presence of $20 \%$ data noise causes visible deterioration to the reconstruction, see Fig. 3(d). Nonetheless, the enhanced reconstruction still exhibits a clear ring shape, and it represents a very good approximation to the true scatterer upon noting the large amount of data noise. 

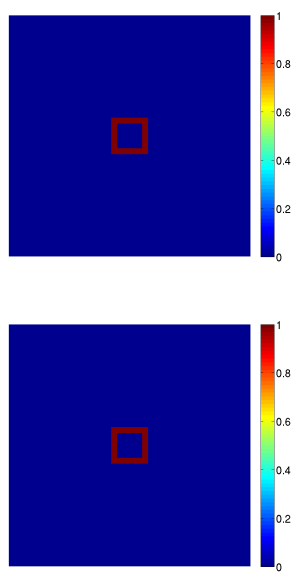

(a) true scatterer
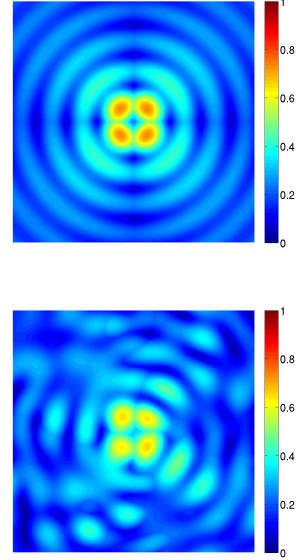

(b) index $\Phi$
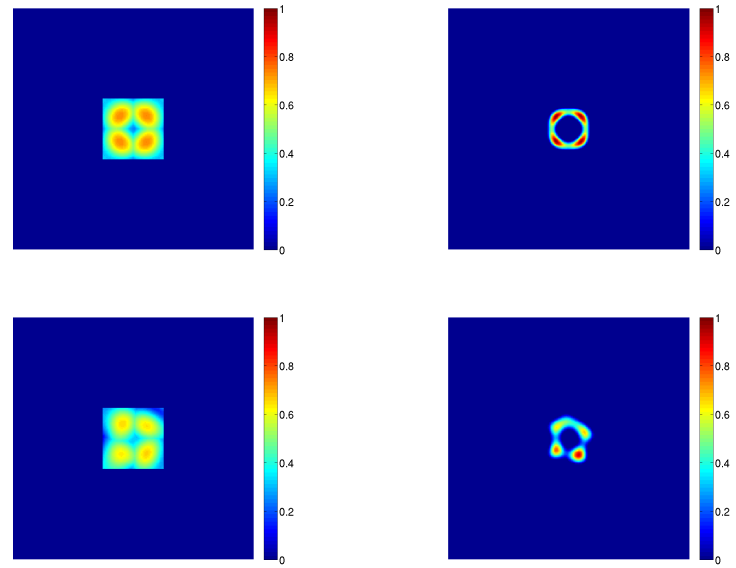

(c) index $\left.\Phi\right|_{D}$

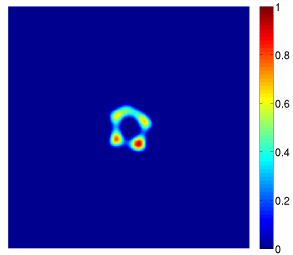

(d) sparse recon.

Figure 3: Numerical results for Example 2: (a) true scatterer, (b) index $\Phi$, (c) index $\left.\Phi\right|_{D}$ (restriction to the subdomain $D$ ) and (d) sparse reconstruction. The first and second rows refer to exact data and the data with $20 \%$ noise, respectively.

\subsection{Three-dimensional example}

Our last example shows the feasibility of the method for three-dimensional problems.

Example 3. We consider two cubic scatterers of width 0.1 centered at $(0.35,0.15,0.15)$ and $(-0.35,0.15,0.15)$, respectively. One single incident field with direction $d=\frac{1}{\sqrt{3}}(1,1,1)^{\mathrm{T}}$ is used, and the coefficient $\eta$ of the scatterers is taken to be 1 .

The scattered field $u^{s}$ is measured at 600 points uniformly distributed on the surface $\Gamma$ of a cubic of width 5 , (i.e., 10 points in each direction). To simulate the scattered field data, we take the sampling domain $\widetilde{\Omega}$ to be the cubic $[-1,1]^{3}$, which is divided into a uniform mesh consisting of small cubes of width $h=0.01$. The inversion domain $D$ for the integral equation (9) is divided into a coarser mesh consisting of small cubes of width 0.03 .

The numerical results for Example 3 with exact data are shown in Fig. 4(b), where each row represents a cross-sectional image along the second coordinate axis $x_{2}$. The scatterer support estimated by the index $\Phi$ agrees reasonably with the exact one, and away from the boundary of the true scatterers, the magnitude of $\Phi$ decreases quickly. However, the reconstructed profile is slightly diffusive in comparison with the exact one, which is reminiscent of the decay property of fundamental solutions. The nonsmooth mixed regularization (10) is carried out on two cubic subregions (of width $0.36 \lambda$ ), cf. Fig. 4(c). Like before, a significant improvement in the resolution is observed: the sparse estimate is much more localized in comparison with the index $\Phi$, and also the magnitude is close to the exact one; see Fig. 4(d). The presence of $20 \%$ data noise does not worsen much the index $\Phi$ and the sparse reconstruction, cf. Fig. 5. Hence the reconstruction algorithm is highly tolerant with respect to data noise.

Lastly, we briefly comment on the computational efficiency of the overall procedure. The first step with the index involves only computing inner products and is embarrassingly cheap and easily parallelized. The accuracy of the support detection is quite satisfactory, and thus a large portion of the sampling domain $\widetilde{\Omega}$ can be pruned from inversion, i.e., $|D| \ll|\widetilde{\Omega}|$. Hence, 
the enhancement via mixed regularization is also rather efficient.

\section{Concluding remarks}

We have presented a novel two-stage inverse scattering method for the inverse medium scattering problem of recovering the refractive index from near-field scattered data. The efficiency and accuracy of the method stem from accurate support detection by the sampling strategy and group sparsity-promoting of the mixed regularization technique. The former is computationally very efficient, and reduces greatly the computational domain for the more expensive inversion via nonsmooth mixed regularization, while the latter achieves an enhanced resolution with the magnitudes and sizes comparable with the exact ones. The numerical results for two- and three-dimensional examples clearly confirm these observations.

These promising experimental results raise a number of interesting questions for further studies. First, the potentials of mixed regularization have been clearly demonstrated. It is of great interest to shed theoretical insights into the model as well as to design efficient acceleration strategies, which for three-dimensional problems remains very challenging. Some partial theoretical results can be found in [14]. Also of much practical relevance is an automated choice of regularization parameters. Second, the reconstructions were obtained with the linearized model, which represents only an approximation to the genuine nonlinear IMSP model. It would be interesting to justify the excellent performance of the linearization procedure. Third, the robustness of the approach to noise is outstanding when compared with more conventional inverse scattering algorithms, especially noting the limited data for inversion. The mechanism of the robustness is not yet clear.

\section{Acknowledgements}

The work of BJ is supported by Award No. KUS-C1-016-04, made by King Abdullah University of Science and Technology (KAUST), and that of JZ is substantially supported by Hong Kong RGC grants (projects 405110 and 404611).

\section{A Numerical method for forward scattering}

We denote by $\mathbb{J}$ the index set of grid points of a uniformly distributed mesh with a mesh size $h>0$ and consider square cells

$$
B_{j}=B_{j_{1}, j_{2}}=\left(x_{j_{1}}^{1}, x_{j_{2}}^{2}\right)+\left[-\frac{h}{2}, \frac{h}{2}\right] \times\left[\frac{h}{2}, \frac{h}{2}\right]
$$

for every tuple $j=\left(j_{1}, j_{2}\right)$ belonging to the index set $\mathbb{J}$. Assume that the domain $\cup_{j \in \mathbb{J}} B_{j}$ contains the scatterer support $\Omega$. We use the mid-point quadrature rule to evaluate the operator $K$, and hence the integral (3) is approximated by

$$
I_{k}-\eta_{k} \sum_{j \in \mathbb{J}} G_{k, j} I_{j} h^{2}=\eta_{k} u^{i n c}\left(x_{k}\right)
$$



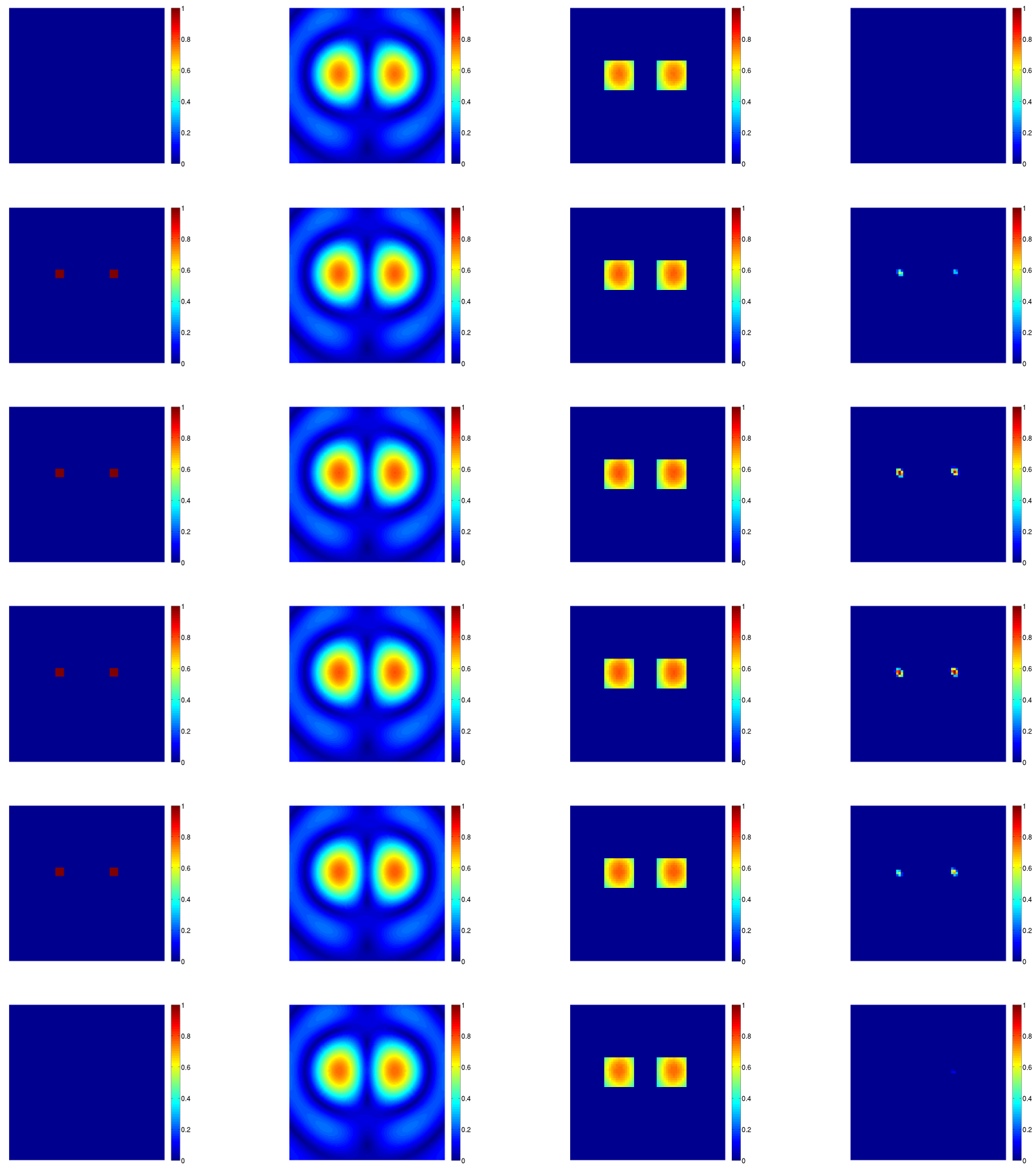

(a) true scatterer

(c) index $\left.\Phi\right|_{D}$

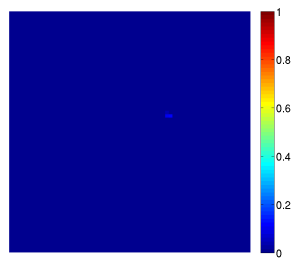

(d) sparse recon.

Figure 4: Numerical results for Example 3 with exact data: (a) true scatter, (b) index $\Phi$, (c) index $\left.\Phi\right|_{D}$ (restriction to the subdomain $D$ ) and (d) sparse reconstruction. From the top to bottom: the cross sectional images at $x_{2}=1.07,1.10,1.13,1.16,1.19$, and 1.22 , respectively. 

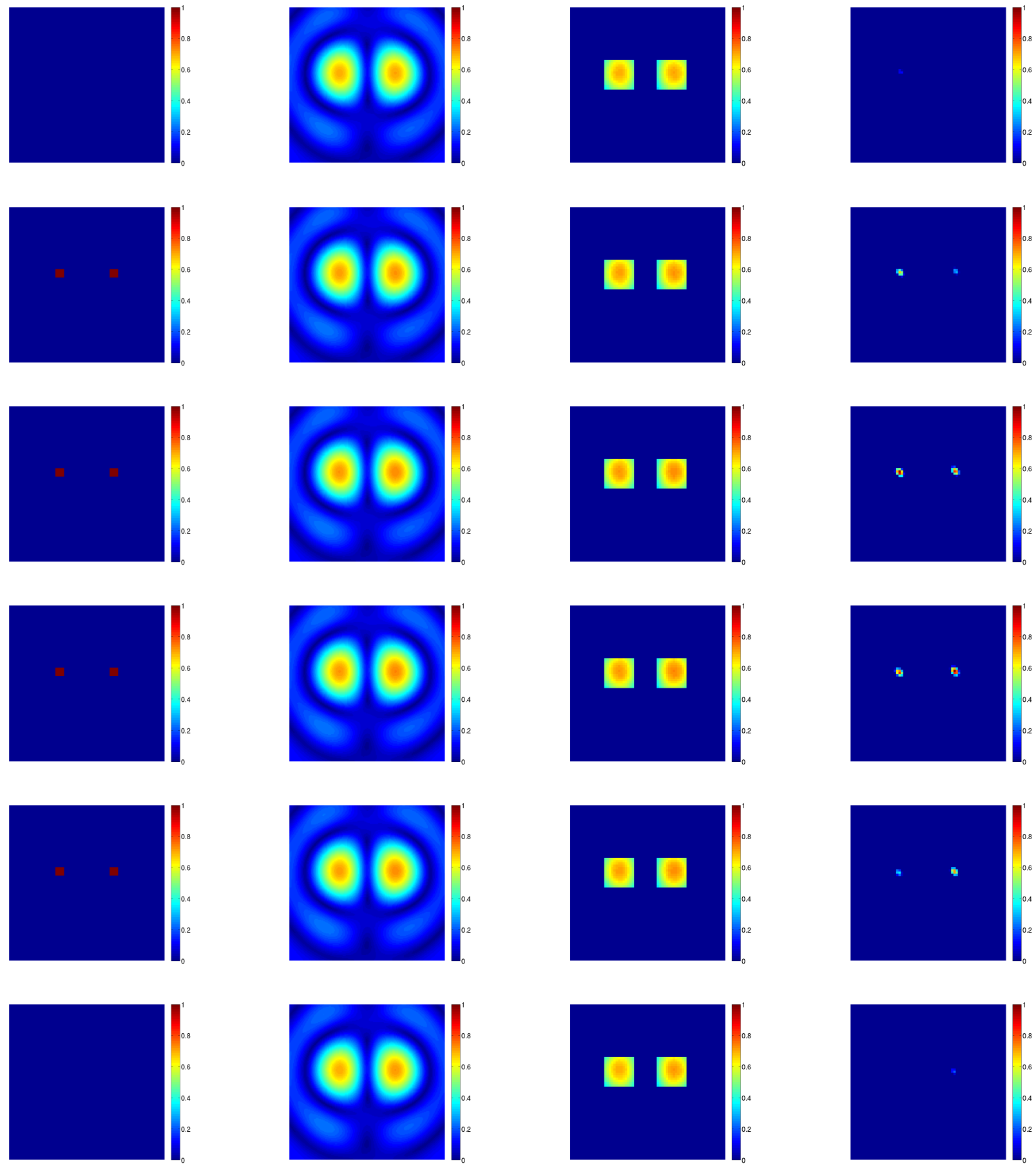

(a) true scatterer

(c) index $\left.\Phi\right|_{D}$

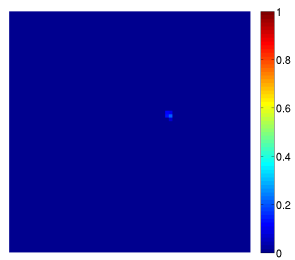

(d) sparse recon.

Figure 5: Numerical results for Example 3 with $20 \%$ noise in the data: (a) true scatter, (b) index $\Phi$, (c) index $\left.\Phi\right|_{D}$ (restriction to the subdomain $D$ ) and (d) sparse reconstruction. From the top to bottom: the cross sectional images at $x_{2}=1.07,1.10,1.13,1.16,1.19$, and 1.22 respectively. 
where $I_{k}=I\left(x_{k}\right)$ and $\eta_{k}=\eta\left(x_{k}\right)$, and the off-diagonal entries $G_{k, j}$ and the diagonal entries $G_{k, k}$ are given by $G_{k, j}=G\left(x_{k}, x_{j}\right)$ and

$$
G_{k, k}=\frac{1}{h^{2}} \int_{\left(-\frac{h}{2}, \frac{h}{2}\right)^{2}} G(x, 0) d x,
$$

respectively. The diagonal entries can be accurately computed by tensor-product Gaussian quadrature rules. The resulting system can be solved using standard numerical solvers, e.g., Gaussian elimination, if the cardinality of the index set $\mathbb{J}$ is medium, and iterative solvers like GMRES. The extension of the procedure to 3D problems is straightforward.

\section{B Semi-smooth Newton method}

In this part, we derive a semi-smooth Newton method for minimizing (10). The optimality condition of the variational problem reads

$$
\left\{\begin{array}{r}
K^{*} K \eta+\alpha \lambda-\beta \Delta \eta-K^{*} u^{s}=0 \\
\lambda-\frac{\lambda+c \eta}{\max (1,|\lambda+c \eta|)}=0
\end{array}\right.
$$

where $\lambda$ is the Lagrange multiplier (dual variable). The second line, the complementarity function, equivalently expresses the inclusion $\lambda \in \partial\|\eta\|_{L^{1}}$, which can be checked directly by pointwise inspection. Thereby, we effectively transforms the inclusion (11) into a numerically amenable nonlinear system. It follows directly from the complementarity relation

$$
\lambda=\frac{\lambda+c \eta}{\max (1,|\lambda+c \eta|)}
$$

that on the active set $\mathcal{A}=\{x \in D:|\lambda+c \eta|(x) \leq 1\}, \eta$ vanishes identically. Otherwise, both the dual variable $\lambda$ and the primal variable $\eta$ need to be solved. We shall solve the system by a semi-smooth Newton method [18]. First observe that the Newton step (with the increments for $\lambda$ and $\eta$ denoted by $\delta \lambda$ and $\delta \eta$, respectively) applied to the following reformulation of equation (13) (on the set $\mathcal{I}=D \backslash \mathcal{A}$ )

$$
\lambda|\lambda+c \eta|-\lambda+c \eta=0
$$

is given by

$$
|\lambda+c \eta| \delta \lambda+\lambda \frac{\lambda+c \eta}{|\lambda+c \eta|}[\delta \lambda+c \delta \eta]-(\delta \lambda+c \delta \eta)+\lambda|\lambda+c \eta|-(\lambda+c \eta)=0,
$$

or equivalently with the notation $\lambda^{+}=\lambda+\delta \lambda$ and $\eta^{+}=\eta+\delta \eta$, we have

$$
\lambda^{+}|\lambda+c \eta|+\lambda \frac{\lambda+c \eta}{|\lambda+c \eta|}\left[\lambda^{+}+c \eta^{+}\right]=\lambda|\lambda+c \eta|+\left[\lambda^{+}+c \eta^{+}\right] .
$$

Next we apply the idea of damping and regularization to the equation and thus get

$$
\lambda^{+}|\lambda+c \eta|+\theta\left[\lambda^{+}+c \eta^{+}\right] \frac{\lambda+c \eta}{|\lambda+c \eta|} \frac{\lambda}{\max (|\lambda|, 1)}=\left[\lambda^{+}+c \eta^{+}\right]+\theta|\lambda+c \eta| \frac{\lambda}{\max (|\lambda|, 1)} .
$$


Here, the purpose of the regularization step $\frac{\lambda}{\max (|\lambda|, 1)}$ is to automatically constrain the dual variable $\lambda$ to $[-1,1]$. The damping factor $\theta$ is automatically selected to achieve the stability. To this end, we let $d=|\lambda+c \eta|, \widetilde{\eta}=d-1, a=\frac{\lambda}{\max (|\lambda|, 1)}$, and $b=\frac{\lambda+c \eta}{|\lambda+c \eta|}$. We arrive at

$$
\lambda^{+}(\widetilde{\eta}+1)+\theta\left[\lambda^{+}+c \eta^{+}\right] a b=\left[\lambda^{+}+c \eta^{+}\right]+\theta a d .
$$

Thus we have

$$
\lambda^{+}=\frac{1}{\widetilde{\eta}+\theta a b}[1-\theta a b] c \eta^{+}+\frac{\theta d}{\widetilde{\eta}+\theta a b} a
$$

To arrive at a simple iteration scheme, we set $\frac{\theta d}{\tilde{\eta}+\theta a b}=1$, i.e., $\theta=\frac{d-1}{d-a b} \leq 1$. Consequently, we obtain a simple iteration

$$
\lambda^{+}=\frac{1-a b}{d-1} c \eta^{+}+\frac{\lambda}{\max (|\lambda|, 1)},
$$

where we have used the relation $\frac{1-\theta a b}{\widetilde{\eta}+\theta a b}=\frac{1-a b}{d-1}$. Substituting this into the first equation gives

$$
K^{*} K \eta^{+}+\alpha c \frac{1-a b}{d-1} \eta^{+}-\beta \Delta \eta^{+}=K^{*} u^{s}-\alpha \frac{\lambda}{\max (|\lambda|, 1)} .
$$

We note that one only needs to solve equation (14) on the inactive set $\mathcal{I}$, since on the active set $\mathcal{A}$, there always holds $\eta^{+}=0$. This has an enormous computational consequence: the size of the linear system in (14) can be very small if $|\mathcal{I}|$ is small, i.e., the solution is sparse. This last relation shows also clearly the sparsity of the solution, and this provides a crispy estimate of the background. Upon obtaining the solution $\eta^{+}$, one can update $\lambda^{+}$on the sets $\mathcal{I}$ and $\mathcal{A}$ according to the second and the first equation, respectively. Lastly, we would like to remark on the consistency of the scheme: if the sequence generated by the semi-smooth Newton method converges, then the limit satisfies the complementarity relation (13) as desired.

\section{References}

[1] A. B. Bakushinsky and K. A. I. Kokurin, M Yu. On stable iterative methods of gradient type for the inverse medium scattering problem. Inv. Probl. Sci. Eng., 13(3):203-218, 2005.

[2] G. Bao and P. Li. Inverse medium scattering for the Helmholtz equation at fixed frequency. Inverse Problems, 21(5):1621-1641, 2005.

[3] F. Cakoni, D. Colton, and P. Monk. The Linear Sampling Method in Inverse Electromagnetic Scattering. SIAM, 2011.

[4] E. J. Candes, J. Romberg, and T. Tao. Robust uncertainty principles: exact signal reconstruction from highly incomplete frequency information. IEEE Trans. Inf. Theory, 52(2):489-509, 2006.

[5] X. Chen and Y. Zhong. MUSIC electromagnetic imaging with enhanced resolution for small inclusions. Inverse Problems, 25(1):015008, 12, 2009.

[6] M. Cheney. The linear sampling method and the MUSIC algorithm. Inverse Problems, 17(4):591-595, 2001. 
[7] D. Colton and A. Kirsch. A simple method for solving inverse scattering problems in the resonance region. Inverse Problems, 12(4):383-393, 1996.

[8] D. Colton and R. Kress. Inverse Acoustic and Electromagnetic Scattering Theory. SpringerVerlag, Berlin, 2 edition, 1998.

[9] I. Daubechies, M. Defrise, and C. De Mol. An iterative thresholding algorithm for linear inverse problems with a sparsity constraint. Comm. Pure Appl. Math., 57(11):1413-1457, 2004 .

[10] A. J. Devaney. Super-resolution processing of multi-static data using time-reversal and music. available at http://www.ece.neu.edu/faculty/devaney/preprints/paper02n_ 00.pdf, 1999.

[11] D. L. Donoho. Compressed sensing. IEEE Trans. Inf. Theory, 52(4):1289-1306, 2006.

[12] T. Hohage. On the numerical solution of a three-dimensional inverse medium scattering problem. Inverse Problems, 17(6):1743-1763, 2001.

[13] T. Hohage. Fast numerical solution of the electromagnetic medium scattering problem and applications to the inverse problem. J. Comput. Phys., 214(1):224-238, 2006.

[14] K. Ito, B. Jin, and T. Takeuchi. Multi-parameter Tikhonov regularization. Methods Appl. Anal., 18(1):31-46, 2011.

[15] K. Ito, B. Jin, and T. Takeuchi. A regularization parameter for nonsmooth Tikhonov regularization. SIAM J. Sci. Comput., 33(3):1415-1438, 2011.

[16] K. Ito, B. Jin, and J. Zou. A direct sampling method to inverse medium scattering problem, 2012 .

[17] K. Ito and K. Kunisch. BV-type regularization methods for convoluted objects with edge, flat and grey scales. Inverse Problems, 16(4):909-928, 2000.

[18] K. Ito and K. Kunisch. Lagrange Multiplier Approach to Variational Problems and Applications. SIAM, Philadelphia, PA, 2008.

[19] B. Jin, D. A. Lorenz, and S. Schiffler. Elastic-net regularization: error estimates and active set methods. Inverse Problems, 25(11):115022 (26pp), 2009.

[20] A. Kirsch. Characterization of the shape of a scattering obstacle using the spectral data of the far field operator. Inverse Problems, 14(6):1489-1512, 1998.

[21] Y. Lu, L. Shen, and Y. Xu. Multi-parameter regularization methods for high-resolution image reconstruction with displacement errors. IEEE Trans. Circ. Syst. I: Reg. Pap., 54(8):1788-1799, 2007.

[22] R. Potthast. A survey on sampling and probe methods for inverse problems. Inverse Problems, 22(2):R1-R47, 2006. 
[23] W. Rachowicz and A. Zdunek. Application of the FEM with adaptivity for electromagnetic inverse medium scattering problems. Comput. Methods Appl. Mech. Engrg., 200(2932):2337-2347, 2011.

[24] R. Schmidt. Multiple emitter location and signal parameter estimation. IEEE Trans. Ant. Prop., 34(3):276-280, 1986.

[25] R. Tibshirani. Regression shrinkage and selection via the lasso. J. Royal Stat. Soc. Ser. B, 58(1):267-288, 1996.

[26] A. N. Tikhonov and V. Y. Arsenin. Solutions of Ill-Posed Problems. John Wiley, New York, 1977.

[27] P. van den Berg, A. L. van Broekhoven, and A. Abubakar. Extended contrast source inversion. Inverse Problems, 15(5):1325-1344, 1999.

[28] M. Vögeler. Reconstruction of the three-dimensional refractive index in electromagnetic scattering by using a propagation-backpropagation method. Inverse Problems, 19(3):739$753,2003$.

[29] S. J. Wright, R. D. Nowak, and M. A. T. Figueiredo. Sparse reconstruction by separable approximation. IEEE Trans. Sig. Process., 57(7):2479-2493, 2009. 